\title{
Plasmodium falciparum clearance with artemisinin-based combination therapy (ACT) in patients with glucose-6-phosphate dehydrogenase deficiency in Mali
}

Abdoulaye K Kone ${ }^{1 *}$, Issaka Sagara ${ }^{1}$, Mahamadou A Thera ${ }^{1}$, Alassane Dicko', Aldiouma Guindo ${ }^{1}$, Seidina Diakite ${ }^{1}$, Joseph Kurantsin-Mills ${ }^{3}$, Abdoulaye Djimde ${ }^{1}$, Asikiya Walcourt², Ogabara Doumbo

\begin{abstract}
Background: Artemisinin-based combination therapy (ACT) is currently the most effective medicine for the treatment of uncomplicated malaria. Artemisinin has previously been shown to increase the clearance of Plasmodium falciparum in malaria patients with haemoglobin E trait, but it did not increase parasite inhibition in an in vitro study using haemoglobin AS erythrocytes. The current study describes the efficacy of artemisinin derivatives on $P$. falciparum clearance in patients with glucose-6-phosphate dehydrogenase deficiency (G6PD), a haemoglobin enzyme deficiency, not yet studied in the same context, but nonetheless is a common in malaria endemic areas, associated with host protection against uncomplicated and severe malaria. The impact of G6PD deficiency on parasite clearance with ACT treatment was compared between G6PD-deficient patients and G6PD-normal group.

Methods: Blood samples from children and adults participants (1 to 70 years old) with uncomplicated $P$. falciparum malaria residing in Kambila, Mali were analysed. Study participants were randomly assigned to receive either artemether-lumefantrine $\left(\right.$ Coartem $^{\oplus}$ ) or artesunate plus mefloquine (Artequin ${ }^{\top M}$ ). A restriction-fragment length polymorphism analysis of PCR-amplified DNA samples was used to identify the (A-) allele of the gene mutation responsible for G6PD deficiency (G6PD*A-). 470 blood samples were thus analysed and of these, DNA was extracted from 315 samples using the QIAamp kit for PCR to identify the G6PD*A- gene.

Results: The DNA amplified from 315 samples using PCR showed that G6PD*A- deficiency was present in 56 participants (17.8\%). The distribution of the specific deficiency was 1\%,7\% and, $9.8 \%$ respectively for homozygous, hemizygous, and heterozygous genotypes. Before treatment, the median parasitaemia and other baseline characteristics (mean haemoglobin, sex and age groups) between G6PD deficiency (hemizygous, heterozygous, and homozygous) and G6PD-normal participants were comparable ( $p>0.05$ ). After treatment, parasite clearance did not change significantly whether the participants were G6PD deficient or G6PD normal on day $1(\mathrm{OR}=1.3 ; \mathrm{Cl}=$ $0.70-2.47 ; \mathrm{p}>0.05)$ and on day $2(\mathrm{OR}=0.859 ; \mathrm{Cl}=0.097-7.61 ; \mathrm{p}>0.05)$.
\end{abstract}

Conclusions: The presence of G6PD deficiency does not appear to significantly influence the clearance of $P$. falciparum in the treatment of uncomplicated malaria using ACT.

\footnotetext{
* Correspondence: fankone@icermali.org

${ }^{1}$ Malaria Research and Training Center, Faculty of Medicine, Pharmacy and

Odonto-stomatology, University of Bamako, P. O. Box 1805 Bamako, Mali

Full list of author information is available at the end of the article
} 


\section{Background}

In a race to combat the ever increasing resistance of Plasmodium falciparum to older anti-malarial drugs, artemisinin, a natural product found in the leafy portions of Artemisia annua (qinghao) and its derivatives, have emerged as alternative drugs for the treatment of falciparum malaria [1]. Artemisinin derivatives are sesquiterpenoides with an endoperoxide, which is the essential component of the anti-malarial activity. With their structural distinction from all other anti-malarial, artemisinins have so far been shown to be effective against multidrug-resistant strains of $P$. falciparum. The use of artemisinin-based combination therapy (ACT) is associated with a rapid clearance of the parasite and a low probability of drug-resistant parasite emergence [2]. ACT is currently recommended by World Health Organization (WHO) for treating uncomplicated falciparum malaria. Several reports evaluating the efficacy of ACT have confirmed its efficacy and safety. Studies by Falade et al in Nigeria showed a 28-day cure rate of about $95 \%$ with artemether-lumefantrine (AL) [3] and 93\% for artesunate-amodiaquine (ASAQ) [4]. Karema et al also reported day 28 cure rates of $95.2 \%$ and $92.0 \%$ for dihydroartemisinin/piperaquine (Artekin) and ASAQ, respectively, in Rwanda [5].

Studies have also shown P. falciparum susceptibility to anti-malarial drugs to correlate with abnormal haemoglobins. A clinical study in Thailand first suggested that haemoglobin E trait (with characteristically increased oxidative activity due to excess $\alpha$-globin chains) interacts with artemisinin derivatives to enhance $P$. falciparum clearance in malaria patients with haemoglobin E trait compared to patients treated with other antimalarials [6]. Another study found a reduced chloroquine and artemisinin efficacy against $P$. falciparum in $\alpha$-thalassemia [7]. Other studies found no differences in parasite susceptibility to chloroquine in $\mathrm{Hb} \mathrm{AS}$ and $\mathrm{Hb}$ AA red blood cells in vitro [8]. More recently, an in vitro study has demonstrated no evidence of elevated artemisinin activity on P. falciparum in haemoglobin AS erythrocytes [9]. These conflicting data on the activity of artemisinin on P. falciparum in abnormal haemoglobin carriers make it imperative to assess the efficacy of ACT in G6PD deficient patients in Mali, where this haemoglobinopathy is common. As far as the literature is concerned, no such studies have been done to study the efficacy of artemisinin against P. falciparum in G6PD deficient patients. G6PD variants correlates with historical distribution of malaria [10] and the mutant allele $(A-)$ encoding G6PD with $10 \%-50 \%$ of abnormal enzyme activity is widespread in Africa [11]. The aim of this study was to determine the clearance of $P$. falciparum and treatment efficacy in uncomplicated malaria as assessed by WHO 28-day protocol using ACT. The study hypothesis was that treatment with ACT may induce faster parasite clearance in G6PD deficient patients compared to G6PD normal patients.

\section{Methods}

\section{Setting and study design}

The study participants were selected from a randomized study conducted in Kambila village. The study participants were randomized to receive artemisinin-based combination therapy artemether-lumefantrine (Coartem $^{\circ}$ ) or artesunate plus mefloquine (Artequin ${ }^{\mathrm{ns}}$ ). The G6PD mutant gene was identified by nested PCR performed on stored blood samples (filter paper dot). The study hypothesis was based on fact that P. falciparum in G6PD deficient patients would clear faster after ACT initiation compared to normal patients. A nested case control design was used to compare G6PD deficient to G6PD-normal subjects with a ratio of one case for four controls.

\section{Study population}

The study participants were selected from children and adults ( 1 to 70 years old) patients who came to the health centre for care during the study period. Only patients with uncomplicated malaria who gave their consent after explanation of study objectives and understood were enrolled into the study. Children were enrolled after obtaining a written parental or guardian fully informed consent. Blood samples were collected from both children and adult patients and were analysed for G6PD status, and parasite clearance since it has been shown that parasite clearance is faster in adults than children [12].

\section{Study site and period}

Study was conducted during a malaria transmission season from August 2004 through January 2005, in Kambila, Mali. Kambila is a peri-urban village of Kati located about $25 \mathrm{~km}$ from Bamako with a population of approximately 1,500 inhabitants. Malaria in this area is hyper-endemic and transmission is highly seasonal. $P$. falciparum is the predominant infecting species, accounting for more than $95 \%$ of malaria cases [13]. Kambila is one of the Mali malaria research sites where malaria drug efficacy data are available, and a prevalence of as high as 17\% G6PD deficiency has also been documented [14].

\section{Sample size}

The sample size was computed based on $70 \%$ of parasite clearance observed 24-hours after artesunate/amodiaquine treatment initiation in a general population at 
Bougoula-Hameau, Sikasso site, a similar malaria hyperendemic area of Mali. Assuming an error risk alpha set at 0.05 , a power of $80 \%$, for one case matched with four controls and fixing a detectable risk estimate at 3.0 (Odds ratio), we will need 55 samples in the cases group (G6PD deficient) and 220 in the control group (non G6PD deficient), rounded up to 56 cases and 259 controls.

\section{Inclusion and exclusion criteria}

Participants were included if they fulfilled the following criteria: 1 ) weighed $\geq 10 \mathrm{~kg}$; 2) had a $P$. falciparum parasite density of between 2,000 and $200,000 / \mu \mathrm{l} ; 3$ ) had an auxiliary temperature $\geq 37.5^{\circ} \mathrm{C} ; 4$ ) were a resident of the study site; and 5) could take medication orally.

Persons were excluded if they had symptoms or signs of severe malaria [15], had a serious disease, had an allergy to one or more study drugs, had used any component of the study drugs within 28 days of enrollment, or were pregnant (detected either clinically or with a urine $ß$-human chorionic gonadotropin test). Community permission and individual written informed consent were provided by a parent or guardian of all participating children, as described by Diallo et al [16]. Withdrawal criteria for the study included (a) protocol violation, (b) participant withdrawal of consent, and (c) development of serious adverse event.

\section{Ethics considerations}

The protocol was reviewed and approved by the ethical committee of the Faculty of Medicine, Pharmacy and Odonto-Stomatology of the University of Bamako before starting the randomized clinical trial. Approval letter from these three Faculty ethical of committees was also obtained before conducting the molecular analysis for the G6PD deficiency study.

\section{Laboratory procedures}

Giemsa-stained thick blood smears were read by experienced laboratory technicians who were blinded to treatment allocations. Parasite densities were calculated by counting the number of asexual parasites until 300 leukocytes were observed and then converting that to parasites per microlitre of blood, assuming an average leukocyte count of $7,500 / \mu \mathrm{l}$. For quality control, $10 \%$ of the slides were selected at random and re-read by another lab technician who was unaware of the results of the first reading.

Two methods were used for DNA extraction on blood spotted filter paper:

(1). An initial extraction was done using methanol. If no DNA could be extracted from blood samples or if the results of the PCR were conflicting, DNA was extracted using the QIAamp kit [17]. (2). If after an initial extraction using methanol yielded no results, or if the results yielded were conflicting, the QIAamp kit [17] was used to extract DNA.

The (A_) allele of the gene responsible for G6PD deficiency was determined by restriction fragment length polymorphism analysis of PCR-amplified DNA samples. Under conditions intended to eliminate the risk of cross-contamination and with appropriate water-only negative controls, exon 4 of G6PD was amplified using a nested PCR protocol: $1 \mu \mathrm{g}$ of genomic DNA was first amplified using primers 5'-GTCTTCTGGGTCAG GGAT-3' (forward) and 5'-GGAGAAAGCTCTCTCT CC-3' (reverse). Denaturation at $94^{\circ} \mathrm{C}$ for 2 min was followed by 45 cycles of denaturation at $94^{\circ} \mathrm{C}$ for $30 \mathrm{~s}$, annealing at $60^{\circ} \mathrm{C}$ for $30 \mathrm{~s}$, extension at $72^{\circ} \mathrm{C}$ for $60 \mathrm{~s}$, and final extension at $72^{\circ} \mathrm{C}$ for $4 \mathrm{~min}$. Nested amplification was performed using primers 5 '-CCTGTTCCCTCTGCCACA-3' (forward) and 5'-GGGGGTCTC AAGAAGTAC-3' (reverse). Denaturation at $94^{\circ} \mathrm{C}$ for 2 min was followed by 35 cycles of denaturation at $94^{\circ} \mathrm{C}$ for $30 \mathrm{~s}$, annealing at $60^{\circ} \mathrm{C}$ for $60 \mathrm{~s}$, extension at $72^{\circ} \mathrm{C}$ for $30 \mathrm{~s}$, and a final extension at $72^{\circ} \mathrm{C}$ for $4 \mathrm{~min}$. Amplification products were recovered with separate pipettes in laboratory areas apart from the PCR-preparation bench and digested with the restriction endonuclease NlaIII [18] or its isoschizomer Hsp92II [19] to detect the G6PD*A mutation at nucleotide position 202. Complete cutting of PCR product identified hemizygous males and homozygous females and cutting of half of the product identified heterozygous females.

\section{Participants surveillance}

Study participants were examined at 1, 2, 3, 7, 14, 21, and 28 days after enrollment or at any time if they did not feel well. A finger skin puncture was used to obtain blood for a thick blood smear and a filter paper dot (for future parasite DNA extraction) at each follow-up visit.

\section{Study endpoints}

The primary study endpoint was the time to parasitaemia disappearance from day 1 after treatment initiation. Parasitaemia clearance was defined as the parasitaemia disappearance at day 1, day 2 and up to day 28 after treatment. Proportion of gametocytes carriers at day 3 was defined as the percent of subjects carrying gametocytes that day regardless of their gametocyte carriage on day 0 . The efficacy of ACT was assessed as an adequate clinical and parasitological response at day 28 according to WHO 2003 guideline [20].

\section{Statistical analysis}

Data were double-entered, validated using Microsoft Access (Microsoft Corporation, Washington, USA), and analysed with STATA version 10.0 (STATA Corporation, 
College Station, TX). Chi-square test or Fisher exact test were used to compare proportions between groups. Mann-Whitney test or Kruskal Wallis test were used as appropriate to assess differences of median parasitaemia between groups for continuous variables. Logistic regression was used to control confounding factors, the odds ratio and exact $95 \%$ CIs were calculated. A $P$ value (twosided $)<0.05$ was considered as statistically significant.

\section{Results}

The DNA amplified from 315 samples out of the 470 samples using PCR showed that G6PD*A- deficiency was present in 56 participants $(17.8 \%)$. The distribution of the specific deficiency was $1 \%, 7 \%$ and, $9.8 \%$ respectively for homozygous, hemizygous, and heterozygous genotypes. The mean haemoglobin and the mean body temperature were compared and no difference was seen between G6PD deficient genotype and normal genotype (p > 0.05) (Table 1).

The G6PD deficiency genotypes were distributed equally between age groups $(\mathrm{p}=0.2)$. The risk estimate of P. falciparum clearance at day 1, for homozygous, hemizygous or heterozygous G6PD deficiency versus G6PD-normal was not significant (Table 2). When median parasitaemia at day 0 for the normal G6PD was compared to the G6PD non-normal in general, there was no difference $(\mathrm{p}=0.1)$.

From day 0 to day 3 the comparison of median parasite density and parasite clearance with respect to the different G6PD genotypes including the normal group didn't show any significant difference between groups (Figure 1).

Data were analysed by age groups (age groups as indicated in table 1) to assess age specific effect on parasite. The study results did not show significant differences between patients with G6PD deficiency compared to patients without the deficiency on day 1. The ACT efficacy treatment on day 28 was similar in the G6PD deficient group compared to the non-deficient group (Table 3 ). Gametocytes carriage at day 3 was not influenced by
Table 2 Risk estimate of $P$. falciparum clearance at day 1, G6PD deficiency genotypes versus G6PD-normal

\begin{tabular}{lccc}
\hline Genotypes of G6PD & $\mathbf{n}$ & OR (95\% IC) & $\mathbf{P}$ \\
\hline Homozygous & & & \\
Hemizygous & 3 & $0.894(0.079-10.12)$ & 0.93 \\
Heterozygous & 22 & $0.842(0.33-2.15)$ & 0.72 \\
Normal & 31 & $0.695(0.303-1.59)$ & 0.40 \\
\hline
\end{tabular}

G6PD deficiency when the G6PD-normal group was compared to G6PD deficient group (Table 4).

\section{Discussion}

The purpose of this study was to determine the combined effect of G6PD deficiency and ACT treatment (artemether-lumefantrine and artesunate plus mefloquine) on parasite clearance in patients with uncomplicated falciparum malaria. Data were analysed by age groups to assess age-specific effect on parasite clearance in patients with G6PD deficiency, since studies have shown that parasite clearance was faster in adults than in children [12,21]. The current study shows no significant difference in parasite clearance between G6PD deficient patients compared to patients without the deficiency when adjusted for age effect.

The overall prevalence of G6PD deficiency was $17.8 \%$ is comparable to other authors finding reported elsewhere in Africa where the prevalence is from $0 \%$ to $25 \%$ [22]. The prevalence of $22.2 \%$ was reported in Congo [23] Genotype frequency was $9.8 \%$ (31/315), 7\% (22/315), and $1 \%$ (3/315) for female heterozygous, male hemizygous and female homozygous respectively. The study findings are similar to those reported in other studies where they also observed genotype frequencies of $12 \%$ (36/303) for female heterozygous, $8 \%$ (25/303) for male hemizygous and $<1 \%(1 / 303)$ for homozygous females [24].

Parasite clearance between the different genotypes at day 0 was not statistically significant as median parasitaemia between abnormal and normal G6PD genotypes

Table 1 Distribution of G6PD deficiency genotypes according to age groups, body temperature and hemoglobin level at enrollment

\begin{tabular}{|c|c|c|c|c|c|c|c|}
\hline \multirow[t]{2}{*}{ G6PD Genotypes } & \multirow{2}{*}{$\begin{array}{l}\text { Temperature } \\
\text { Mean }{ }^{\circ} \mathrm{C} \text { (Range) }\end{array}$} & \multirow{2}{*}{$\begin{array}{c}\text { Haemoglobin } \\
\text { Hb/dl } \\
\text { Mean (Range) }\end{array}$} & \multicolumn{5}{|c|}{ Age groups } \\
\hline & & & $\begin{array}{c}<5 \text { years } \\
n(\%)\end{array}$ & $\begin{array}{c}\text { 5-10 years } \\
\text { n (\%) }\end{array}$ & $\begin{array}{c}\text { 11-17 years } \\
\text { n (\%) }\end{array}$ & $\begin{array}{c}>17 \text { years } \\
n(\%)\end{array}$ & $\begin{array}{c}\text { Total Number } \\
\text { n (\%) }\end{array}$ \\
\hline Homozygous & $38.0(0.8)$ & $11.4(1.6)$ & $1(0,9)$ & $2(1,3)$ & $0(0)$ & $0(0)$ & $3(1)$ \\
\hline Hemizygous & $38.5(2.8)$ & $11.2(5.3)$ & $5(4.7)$ & $13(8.7)$ & $3(6,1)$ & $1(10)$ & $22(7)$ \\
\hline Heterozygous & $38.5(2.3)$ & $11.4(8.0)$ & $6(5,6)$ & $14(9,4)$ & $9(18,4)$ & $2(20)$ & $31(9,8)$ \\
\hline Normal & $38.5(2.9)$ & $11.2(9.1)$ & 95 (88.8) & $120(80.5)$ & $37(75.5)$ & $7(70.0)$ & $259(82,2)$ \\
\hline Total & $38.5(2.9)$ & $11.2(9.1)$ & 107 (100) & 149 (100) & 49 (100) & $10(100)$ & 315 (100) \\
\hline
\end{tabular}

Fisher exact test $p=0.2$ for G6PD Genotypes within age groups.

ANOVA $p \geq 0.05$ for G6PD Genotypes and body temperature mean or haemoglobin mean. 


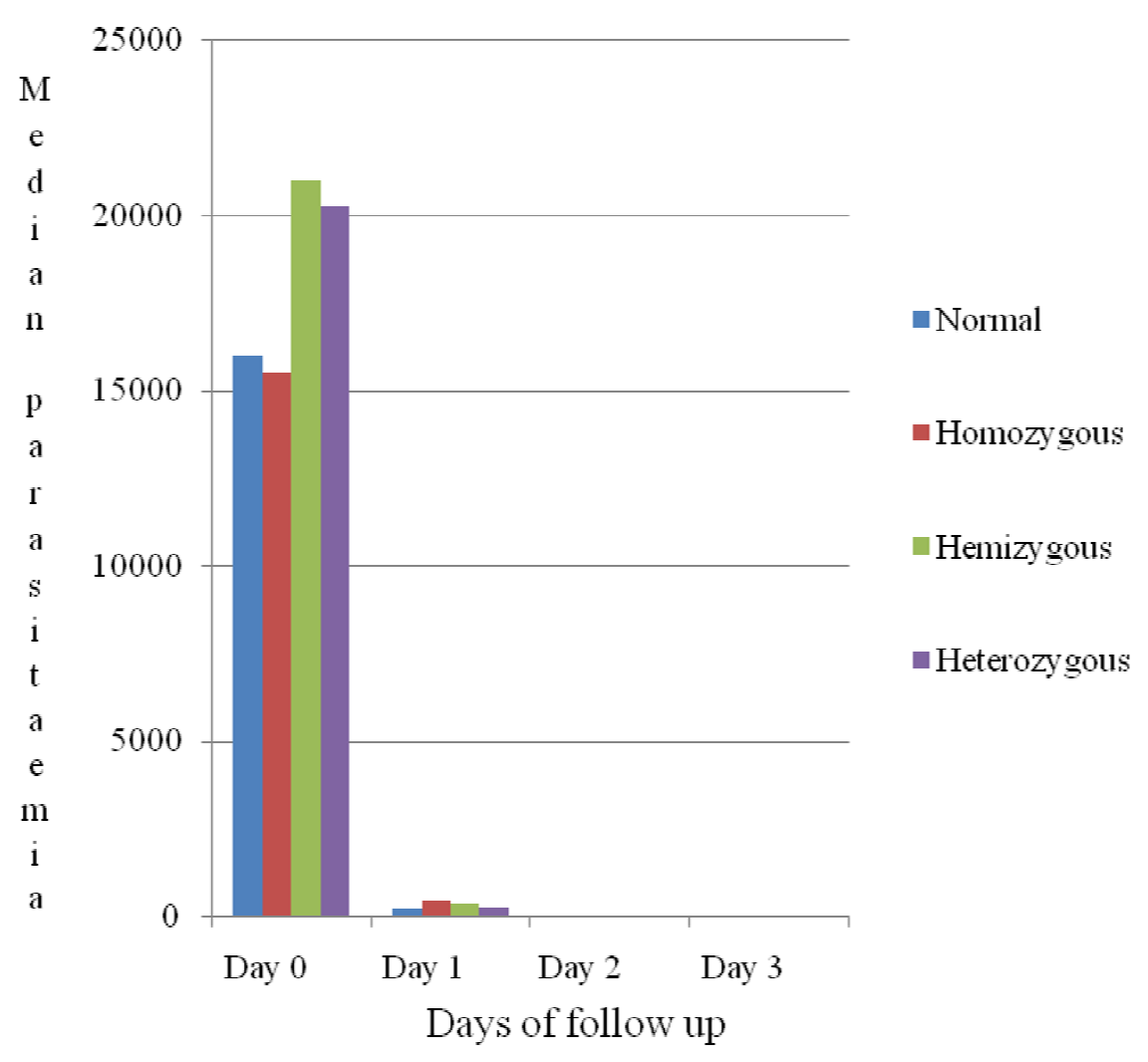

Figure 1 Clearance and density of $P$. falciparum from day 0-day 3, G6PD deficiency genotypes versus G6PD-normal.

was not different. The study results have shown that hemizygous and heterozygous had a tendency of elevated median parasitaemia. Similar observation with geometric mean parasitaemia was also reported elsewhere [24].

A comparison of the median parasitaemia at day 0 between normal G6PD patients and G6PD deficient patients in general showed no difference (median parasite of 20212 parasites/ $\mu$ l versus 15975 parasites/ $\mu 1$ respectively; $\mathrm{p}=0.1$ ). The study results did not confirm findings by Nkuo-Akenji et al., 2004 who showed a significant difference in the mean parasite density in G6PD

Table 3 Association between G6PD deficiency and 28-day follow-up PCR corrected ACT efficacy

\begin{tabular}{lccccc}
\hline Efficacy & $\begin{array}{c}\text { Hemizygous } \\
\mathbf{n}(\%)\end{array}$ & $\begin{array}{c}\text { Heterozygous } \\
\mathbf{n}(\%)\end{array}$ & $\begin{array}{c}\text { Homozygous } \\
\mathbf{n}(\%)\end{array}$ & $\begin{array}{c}\text { Normal } \\
\mathbf{n}(\%)\end{array}$ & $\begin{array}{c}\text { Total } \\
\mathbf{n}(\%)\end{array}$ \\
\hline Cured* & $20(100)$ & $29(96.6)$ & $3(100.0)$ & 237 & 299 \\
& & & & $(98.02)$ & $(98.03)$ \\
$\begin{array}{l}\text { Failure } \\
\text { cases }\end{array}$ & $0(0.0)$ & $1(3.33)$ & $0(0.0)$ & $5(1.98)$ & 6 \\
\hline Total & $20(100)$ & $30(100)$ & $3(100)$ & 252 & 305 \\
& & & & $(100)$ & $(100)$
\end{tabular}

${ }^{*}$ Adequate Clinical and Parasitological Response

Fisher exact test $=0.685$ enzyme deficient group compared to the G6PD normal group. That study found a mean parasite density of 3.7 $( \pm 3.9, \mathrm{SD})$ for enzyme deficient individuals and $4.4( \pm$ 5.0, SD) for enzyme active individuals $(\mathrm{p}<0.05)$ [25].

The study results have shown that G6PD deficiency had no significant association with parasite clearance and median parasitaemia from day 0 to day 3 (Figure 1). The detectable risk estimate of three-fold parasite clearance used to calculate the sample size in this study may also be too high risk estimate, which may have resulted in a small simple size and, therefore, underpowered the study to detect any small differences in parasite clearance that

Table 4 Comparison of proportions of gametocytes carriers at day 3, G6PD deficient patients versus G6PDnormal patients

\begin{tabular}{lccc}
\hline \multirow{2}{*}{ G6PD Status } & \multicolumn{3}{c}{ Proportion of gametocytes carriers } \\
\cline { 2 - 4 } & Gametocytes (-) & Gametocytes (+) & Total \\
\cline { 2 - 4 } & $\mathbf{n}(\%)$ & $\mathbf{n}(\%)$ & $\mathbf{n}$ \\
\hline Deficiency & $54(96.4)$ & $2^{*}(3.6)$ & 56 \\
Normal & $252(97.7)$ & $6(2.3)$ & 258 \\
\hline Total & $306(97.5)$ & $8(2.5)$ & 314
\end{tabular}

Fisher exact test $\mathrm{p}=0.63$

*The two cases occurred in heterozygous genotype deficiency 
may have been observed between deficient and non deficient G6PD patients on day 1 or day 2.

Other factors such as the molecular biology methods used to determine G6PD deficiency used in our study may also undermine the role of G6PD deficiency in parasite clearance. Enzyme assay appears to better reflect the biological effects of the G6PD enzyme more than genotyping. This was suggested in a recent study which showed that among female G6PD-deficient patients, but not male patients, whose genotype was also confirmed by enzymatic methods to have low enzyme activity as shown by enzyme assay were significantly protected from uncomplicated malaria, while patients with higher levels of enzyme activity were not protected against uncomplicated malaria [26].

No significant association between ACT efficacy and G6PD deficiency was seen in our data (Table 4). Although host immune response could play a role in treatment efficacy as other studies have demonstrated [27], the fast parasite clearance and treatment efficacy observed in this study regardless of the G6PD deficiency status is similar to what has been observed in other ACT trials in Mali [13,28]. This is indicative of the high efficacy rates in general of the ACT in Mali.

The possibility of reduced susceptibility of parasites to ACT in G6PD deficient patients could not also be ruled out since in a study with $\alpha$-thalassemia patients, it was hypothesized that the infected variant erythrocytes could not accumulate as much drug as infected normal erythrocytes $(7,9)$. Furthermore, the authors had shown that the reduction in artemisinin accumulation in infected variant erythrocytes was due partly to competition with uninfected erythrocytes for the drug, and partly to the lower drug accumulation inside the parasite $[7,29]$. Therefore, a possible lower drug accumulation in parasites in G6PD deficient red cells could be a disadvantage to G6PD-deficient carriers. This effect is however, yet to be demonstrated in vitro studies.

In contrast another study reported that among hemoglobin $\mathrm{E}$ patients treated with artemisinin derivatives, the presence of hemoglobin $E$ trait was associated with an estimated 2.9-fold increase in parasite clearance rate (95\% CI, 1.4-6.3; $P=.006$ ) [6].

The present study looked at the role of G6PD deficiency in gametocyte carriage and found that the proportion of gametocytes carried was similar in G6PDdeficient and G6PD-normal individuals after treatment at day 3. Similar percentage of gametocyte carriage has also been shown with ACT in the general population in Mali [13,28].

The clearance of $P$. falciparum using ACT in G6PDdeficient patients with uncomplicated malaria may require both enzymatic assay and genetic analysis of the mutation to detect and characterize G6PD deficiency. In clinical trials, whilst enzymatic assays seem to more closely approximate biologic function and correlate with protection, genetic analysis may uncover novel variants causing disease and thereby provide important insights for treatment of uncomplicated malaria.

The likely explanation for a lack of difference in parasite clearance time between those with and without G6PD deficiency is simply that clearance was rapid in all groups due to the rapid activity of artemisinin derivative, and that, if there was any difference, it could not be discriminated with once-daily assessment.

\section{Conclusions}

The study findings suggest that G6PD deficiency did not increase or reduce the parasite clearance and the efficacy of ACT. The biological assays that detect G6PD enzyme activity during malaria treatment may reflect the level of G6PD deficiency and, therefore, may more accurately assess parasite clearance in the presence of treatment rather than using PCR methodology to determine G6PD deficiency as in this study. Other confounding factors such as other host erythrocyte variants and host relative immunity may also play a crucial role in G6PD activity. An in vitro study and in vivo with several daily assessments may be help to understand the interaction between parasite clearance, ACT and G6PD deficiency.

\section{Acknowledgements}

We would like to thank all study volunteers for participating in this study; the staff of the Malaria Research and Training Center, Department of Epidemiology of Parasitic Diseases, the Faculties of Medicine, Pharmacy and Odonto-Stomatology of the University of Bamako, Mali and the College of Medicine at Howard University.

This study was supported through a Malaria Training Grant (NIH/FIC \#D43TW-01589) handled by University of Maryland (USA), BMP/MRTC/DEAP/ FMPOS (Mali) and by Pharmatech Inc. (Denver, CO). Our thanks also to the local West African French representative of Mepha Ltd. Pharmatech, Mr Aliou Adamonwho donated the AS + MEF used in the study while conducting ACTs efficacy study in Mali.

We are grateful to Chistopher V. Plove, Nicole Eddington from University of Maryland (USA) for providing reagents supported by Malaria Training Grant. We are also grateful to Dr. Asikiya Walcourt of Howard University College of Medicine, Washington DC for supplying part of the reagents used for the G6PD molecular analysis in this study. Dr. Walcourt was supported by a grant from NIH/NIGMS (3S06GM08016-39S1). The funding agencies had no role in study design, data collection and analysis, preparation of the manuscript, or the decision to publish the study.

\section{Author details}

${ }^{1}$ Malaria Research and Training Center, Faculty of Medicine, Pharmacy and Odonto-stomatology, University of Bamako, P. O. Box 1805 Bamako, Mali. ${ }^{2}$ Department of Physiology \& Biophysics, Howard University College of Medicine, 520 W Street, Rm 413, Seely G. Mudd Building, NW, Washington, DC 20059, USA. ${ }^{3}$ Departments of Medicine, Pharmacology \& Physiology, The George Washington University Medical Center, Washington, DC 20037, USA.

\section{Authors' contributions}

IS, MAT, AD, AKK, AW, JK-M and OKD participated in study design. IS, AKK and MTA contributed in data management and analysis. AKK, SD, and AG did the molecular analysis in determining the status of G6PD deficiency. All authors participated in the preparation of the manuscript and approved the final version. 


\section{Competing interests}

The authors declare that they have no competing interests.

Received: 19 July 2010 Accepted: 21 November 2010

Published: 21 November 2010

\section{References}

1. Klayman DL: Qinghaosu (artemisinin): an antimalarial drug from China. Science 1985, 228:1049-1055.

2. White NJ: Delaying antimalarial drug resistance with combination chemotherapy. Parassitologia 1999, 41:301-308.

3. Falade C, Makanga M, Premji Z, Ortmann CE, Stockmeyer M, de Palacios Pl: Efficacy and safety of artemetherlumefantrine (Coartem) tablets (sixdose regimen) in African infants and children with acute, uncomplicated falciparum malaria. Trans R Soc Trop Med Hyg 2005, 99:459-467.

4. Falade CO, Ogundele AO, Yusuf BO, Ademowo OG, Ladipo SM: High efficacy of two artemisinin-based combinations (artemether/ lumefantrine and artesunate plus amodiaquine) for acute uncomplicated malaria in Ibadan, Nigeria. Trop Med Int Health 2008, 13:635-643.

5. Karema C, Fanello Cl, van Overmeir C, van Geertruyden JP, van Doren W, Ngamije D, D'Alessandro U: Safety and efficacy of dihydroartemisinin/ piperaquine (Artekin) for the treatment of uncomplicated Plasmodium falciparum malaria in Rwandan children. Trans R Soc Trop Med Hyg 2006, 100:1105-1111.

6. Hutagalung R, Wilairatana P, Looareesuwan S, Brittenham GM, Gordeuk VR Influence of hemoglobin $\mathrm{E}$ trait on the antimalarial effect of artemisinin derivatives. JID 2000, 181:1513-1516.

7. Yuthavong Y, Butthep P, Bunyaratvej A, Fucharoen S: Decreased sensitivity to artesunate and chloroquine of Plasmodium falciparum infecting hemoglobin $\mathrm{H}$ and/or hemoglobin Constant Spring erythrocytes. J Clin Invest 1989, 83:502-505.

8. Nguyen-Dinh P, Parvin RM: Haemoglobin $S$ and in vitro chloroquine susceptibility of Plasmodium falciparum. Lancet 1986, 2:1278.

9. Allison AC: Glucose-6-phosphate dehydrogenase deficiency in red blood cells of East Africans. Nature 1960, 186:531-532.

10. Beutler E, Kuhl W, Vives-Corrons JL, Prchal JT: Molecular heterogeneity of glucose-6-phosphate dehydrogenase A. Blood 1989, 74:2550-2555.

11. Walcourt A, Kurantsin-Mills J, Adenuga BB, Loyevsky M, Kassim OO, Gordeuk VR: Plasmodium falciparum: Activity of artemisinin against Plasmodium falciparum cultured in sickle trait hemoglobin AS and normal hemoglobin AA red blood cells. Expl Parasitol 2008, 120:381-384.

12. Rogier C: Natural history of Plasmodium falciparum malaria and determining factors of the acquisition of antimalaria immunity in two endemic areas, Dielmo and Ndiop (Senegal). Bull Mem Acad R Med Belg 2000, 155:218-226.

13. Sagara I, Diallo A, Kone M, Coulibaly, Diawara SI, Guindo O, Maiga H, Mohamed BM, Sissoko M, Dicko A, Djimde A, Doumbo OK: A randomized trial of artesunate-mefloquine versus artemether-lumefantrine for treatment of uncomplicated Plasmodium falciparum Malaria in Mali. Am J Trop Med Hyg 2008, 79:655-661.

14. Crompton PD, Traore B, Kayentao K, Doumbo S, Ongoiba A, Diakite SA, Krause MA, Doumtabe D, Kone $Y$, Weiss G, Huang CY, Doumbia S, Guindo A, Fairhurst RM, Miller LH, Pierce SK, Doumbo OK: Sickle cell trait is associated with a delayed onset of malaria: implications for time-toevent analysis in clinical studies of malaria. J Infect Dis 2008, 198:1265-1275.

15. World Health Organization: Communicable disease cluster. Severe falciparum malaria. Trans R Soc Trop Med Hyg 2000, 94:S1-S90.

16. Diallo DA, Doumbo OK, Plowe CV, Wellems TE, Emanuel EJ, Hurst SA: Community permission for medical research in developing countries. Clinical Infect Dis 2005, 41:255-259.

17. Website title [Qiagen]. [http://www.qiagen.com]

18. Website title NEB. [http://www.neb.com].

19. Website title [Promega]. [http://www.promega.com]

20. WHO: Assessment and monitoring of antimalarial drug efficacy for the treatment of uncomplicated Falciparum malaria. WHO/HTM/RBM/2003.50

21. Tripathy V, Reddy BM: Present status of understanding on the G6PD deficiency and natural selection. J Postgrad Med 2007, 53:193-202.

22. Luzzatto L, Mehta A: Human erythrocyte glucose-6-phosphate dehydrogenase deficiency. In The metabolic basis of inherited disease. 6 edition. Edited by: Scriver CR, Baudet AL, Sly WS, Valle D. McGraw-Hill, New York; 1989:2237-2265.

23. Bouanga JC, Mouélé R, Préhub C, Wajcmanb H, Feingold J, Galactéros F: Glucose-6-phosphate dehydrogenase deficiency and homozygous sickle cell disease in Congo. Hum Hered 1998, 48:192-197.

24. Parikh S, Dorsey G, Rosenthal PJ: Host polymorphisms and the incidence of malaria in Ugandan children. Am J Trop Med Hyg 2004, 71:750-753.

25. Nkuo-Akenji TK, Wepngong P, Akoachere JF: Effects of ABO/Rh blood groups, G-6-P-D enzyme activity and haemoglobin genotypes on malaria parasitaemia and parasite density. Afr J Health Sci 2004, 11:93-97.

26. Johnson MK, Clark TD, Njama-Meya D, Rosenthal PJ, Parikh S: Impact of the method of G6PD deficiency assessment on genetic association studies of malaria susceptibility. PLoS One 2009, 4:e7246, PubMed PMID: 19789650; PubMed Central PMCID: PMC2748715.

27. Mayxay M, Chotivanich K, Pukrittayakamee S, Newton P, Looareesuwan S, White NJ: Contribution of humoral immunity to the therapeutic response in falciparum malaria. Am J Trop Med Hyg 2001, 65:918-923.

28. Sagara I, Rulisa S, Mbacham W, Adam I, Sissoko K, Maiga H, Traore OB, Dara N, Dicko YT, Dicko A, Djimdé A, Jansen FH, Doumbo OK: Efficacy and safety of a fixed dose artesunate-sulphamethoxypyrazine-pyrimethamine compared to artemether-lumefantrine for the treatment of uncomplicated falciparum malaria across Africa: a randomized multicentre trial. Malar J 2009, 8:63.

29. Kamchonwongpaisan S, Chandra-ngam G, Avery MA, Yuthavong Y: Resistance to artemisinin of malaria parasites (Plasmodium falciparum) infecting a-thalassemic erythrocytes in vitro: competition in drug accumulation with uninfected erythrocytes. J Clin Invest 1994, 93:467-473.

doi:10.1186/1475-2875-9-332

Cite this article as: Kone et al:: Plasmodium falciparum clearance with artemisinin-based combination therapy (ACT) in patients with glucose6-phosphate dehydrogenase deficiency in Mali. Malaria Journal 2010 9:332.

\section{Submit your next manuscript to BioMed Central and take full advantage of:}

- Convenient online submission

- Thorough peer review

- No space constraints or color figure charges

- Immediate publication on acceptance

- Inclusion in PubMed, CAS, Scopus and Google Scholar

- Research which is freely available for redistribution
C Biomed Central 